\title{
DETERMINACIÓN DE VARIABLES CUALITATIVASCOMO FACTORES CLAVES EN LA PROGRAMACIÓNDE LOS PROYECTOS DE EDIFICIOS EN ALTURA, REGIÓN COQUIMBO, CHILE
}

\author{
Determination of qualitative variables as key factors in the programming \\ of building projects In altura, Coquimbo region, Chile
}

\section{EPISTEMUS}

ISSN: 2007-8196 (electrónico)

ISSN: 2007-4530 (impresa)

MSC. Ing. Oscar Contreras ${ }^{1}$

Ing. Ronnie Kauer ${ }^{2}$

Recibido: 12 de febrero de 2017

Aceptado: 27 de julio de 2017

Autor de Correspondencia:

MSC. Ing. Oscar Contreras

Correo: ocontreras@userena.cl

\section{Resumen}

En el presente trabajo se realiza un análisis y se determina en términos cualitativos los factores claves que inciden en la adecuada programación de las duraciones de las actividades en los proyectos de construcción de edificios en altura en la ciudad de Coquimbo, Chile. El análisis profundiza el estudio y desarrollo del modelo estocástico pert/cpm modificado aplicado con antelación, del cual nace la búsqueda de explicaciones de las desviaciones obtenidas, encontrándose, variables del tipo cualitativas que influyen en el resultado de dichas desviaciones al realizar un proyecto de la envergadura del indicado. Estas variables cualitativas, se definen entonces como factores claves, proponiéndose un modelo para con su consideración en los mencionados proyectos de edificación.

Palabras clave: programación; proyectos; gestión; estocasticidad

\section{Abstract}

In this paper, an analysis and determined in qualitative terms the key factors that affect the proper programming of the durations of activities in construction projects rise buildings in the city of Coquimbo, Chile. The analysis explores the study and development of the stochastic model pert/cpm applied modified in advance, from which springs the search for explanations of the deviations obtained, being among others, kind of qualitative variables that influence the outcome of these deviations, to conduct a project the size indicated. These qualitative variables are then defined as key factors in proposing a model for consideration in the said building projects.

Keywords: programming; Projects; management; stochasticity 


\section{INTRODUCCIÓN}

A pesar de buscar con distintas técnicas numéricas mayor precisión en la programación y secuenciación de las actividades de un proyecto, como por ejemplo redes neuronales con respecto a lo realizado, aún persisten diferencias que presentan cierta importancia significativa a los dueños o inversionistas de los proyectos, sobre todo por la limitación de recursos [1].

En la Región de Coquimbo, una zona semi-desértica con un gran potencial de crecimiento y desarrollo de la urbanización en donde su cercanía a los centros mineros importantes de la Región de Atacama y Antofagasta, así como la tranquilidad, zona turística y moderado costo de vida con respecto a las zonas anteriormente indicadas sea un atractivo para que gran cantidad de personas opten por vivir y/o vacacionar en la zona ha generado un fuerte impulso en la industria de la construcción en los últimos años. En la Tabla 1 se puede apreciar por medio del indicador Inacor, que (con excepción de las regiones de Antofagasta y Aysén), es la región con mayor crecimiento a nivel nacional.

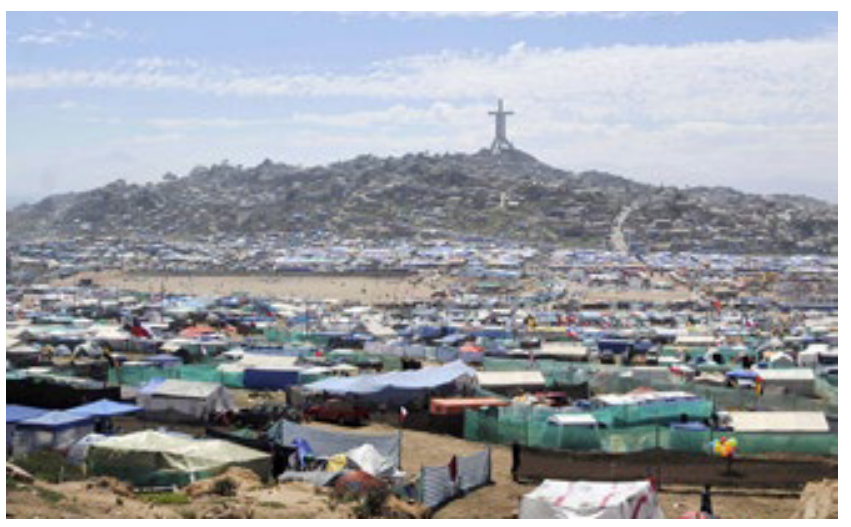

Tabla 1. INACOR, 2013

ÍNDICE DE ACTIVIDAD DE LA CONSTRUCCIÓN REGIONAL Agosto 2013 (Variación mensual, en \%)

\begin{tabular}{|c|c|c|c|c|}
\hline & Indi & cadores Parciale: & & \\
\hline & $\begin{array}{l}\text { Empleo } \\
\text { Sectorial }\end{array}$ & $\begin{array}{l}\text { Permisos de } \\
\text { Edificación }\end{array}$ & $\begin{array}{c}\text { Consumo de } \\
\text { Cemento }\end{array}$ & INACOR \\
\hline XV Arica y Parinacota & $-36,6$ & $-34,5$ & $-23,8$ & $-7,0$ \\
\hline | Tarapacá & $-1,4$ & $-53,5$ & $-6,6$ & $-6,8$ \\
\hline || Antofagasta & $-7,4$ & $-16,1$ & 41,2 & 21,8 \\
\hline III Atacama & $-0,8$ & $-29,5$ & $-12,7$ & $-5,0$ \\
\hline IV Coquimbo & $-1,1$ & 59,8 & 11,5 & 13,4 \\
\hline V Valparaíso & $-13,5$ & 66,9 & 9,1 & 4,6 \\
\hline V| O'Higgins & $-3,0$ & $-57,8$ & 10,4 & 5,3 \\
\hline VII Maule & $-0,5$ & 68,7 & $-3,2$ & 1,1 \\
\hline VIII Bío Bio & 3,1 & 31,0 & $-11,0$ & $-7,4$ \\
\hline IX La Araucanía & 19,9 & 50,8 & 4,1 & 12,7 \\
\hline XIV Los Ríos & $-15,5$ & 92,7 & $-34,8$ & 0,5 \\
\hline X Los Lagos & 12,8 & 27,6 & $-13,9$ & 5,7 \\
\hline XI Aysén & 17,2 & 75,3 & $-46,4$ & 17,0 \\
\hline XII Magallanes & $-8,0$ & $-78,8$ & 49,6 & 8,7 \\
\hline RM. Metropolitana & 1,3 & $-39,2$ & 8,6 & 4,1 \\
\hline
\end{tabular}

Nota: Los crecimientos corresponden a las series en promedio móviles de tres meses desestacionalizadas.

Fuente: $\mathrm{CChC}$ en base a las estadísticas del INE e ICH.

En la Tabla 2 se muestra el PIB regional en donde se aprecia el crecimiento del rubro de la industria de la construcción en la zona.

Tabla 2. PIB por Actividad Económica, Región de Coquimbo

\begin{tabular}{|c|c|c|c|c|c|c|c|}
\hline Actividad & 2003 & 2004 & 2005 & 2006 & 2007 & 2008 & 2009 (1) \\
\hline Pesca & 25.746 & 23.958 & 18.006 & 23.883 & 24.914 & 33.415 & 31.383 \\
\hline Minería & 218.175 & 228.872 & 209.176 & 212.899 & 204.596 & 235.198 & 212.193 \\
\hline Industria Manufacturera & 68.462 & 75.174 & 77.013 & 74.938 & 77.732 & 84.493 & 82.172 \\
\hline Electricidad, Gas y A gua & 37.519 & 30.277 & 34.398 & 34.403 & 30.762 & 27.593 & 36438 \\
\hline Transporte y Comunicaciones & 114.485 & 120.540 & 124.268 & 127.427 & 131.945 & 133.38 & 134.773 \\
\hline Servicios Financieros y Empresariales (2) & 93.376 & 99.917 & 108.391 & 119.339 & 132.998 & 134.981 & 134.566 \\
\hline Propiedad de Vivienda & 92.802 & 95.302 & 98.546 & 102.247 & 106.526 & 110.970 & 115.182 \\
\hline Servicios Personales (3) & 171.512 & 181.390 & 190.487 & 201.638 & 206.031 & 216.566 & 220.848 \\
\hline A dminis tración Pública & 66.825 & 68.891 & 70.896 & 72.909 & 75.862 & 78.689 & 80.876 \\
\hline Menos: Imputaciones Bancarias & -20.429 & -21.269 & -25.403 & -24.686 & -28.754 & -29.930 & -30.206 \\
\hline
\end{tabular}

(1) Cifras provisionales.

(2) Incluye servicios financieros, seguros, arriendo de inmuebles y servicios prestados a empresas.

(3) Incluye educación y salud -pública y privada- y otros servicios.

Fuente: Banco Central de Chile, 2011 


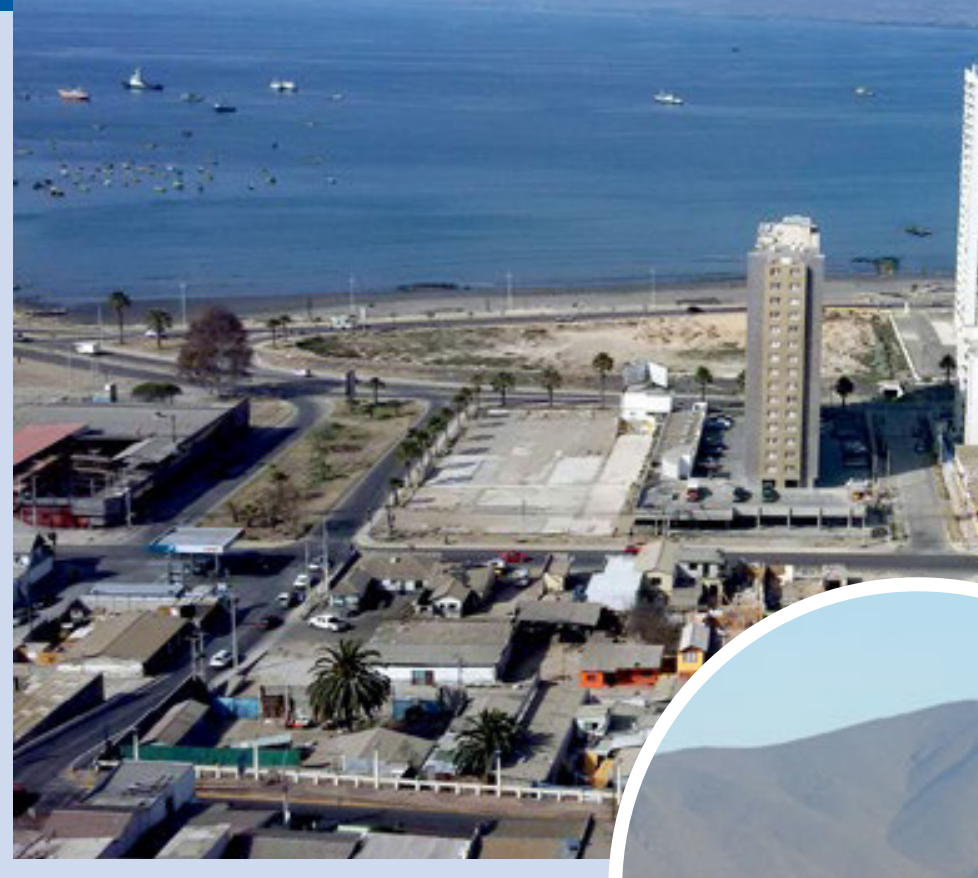

Para subsanar en parte el desarrollo urbanístico en el borde costero, debido a la geografía circundante, no se inicia el desarrollo de la construcción de edificios en altura, los cuales superan los $18-20$ pisos. Una de las problemáticas encontradas en la construcción de los edificios de altura en la región de Coquimbo es el manejo operacional de la programación de proyectos (6), la que con un $95 \%$ se realiza con cartas Gantt, en donde solo se considera la duración de las actividades en términos determinísticos. En estudios anteriores del mismo autor [2], se propuso un Modelo de Programación Pert/Cpm Modificado, el que incorporando el concepto de la estocasticidad, así como el manejo de tecnologías de la informática actuales, nos permitió acercarnos a mitigar esa diferencia entre lo real y lo programado. Como resultado se le entrega a los dueños del proyecto o inversionistas el concepto del riesgo y de la respectiva variabilidad a la que están expuestas las programaciones de las duraciones de las actividades y de los costos asociados del proyecto. Sin embargo, del análisis de los resultados, tanto de los modelos tradicionales como Carta Gantt, así como Pert/Cpm [3]; [4] y Pert/Cpm Modificado [2]. Según los resultados obtenidos, es claro que persisten diferencias significativas.

\section{DETERMINACIÓN DEL FACTOR K DE AJUSTE EN BASE A VARIABLES EXTERNAS}

En la mayoría de los casos analizados en el ámbito de nuestro estudio, por no decir en su totalidad, no se alcanza el desarrollo de la obra con los tiempos y costes programados con los métodos de programación conocidos. Sólo con los métodos estocásticos se logra alcanzar valores entre $7 \%$ y $10 \%$ de precisión tanto en la duración como en los costos del proyecto.

De lo anterior es donde nos damos cuenta de que

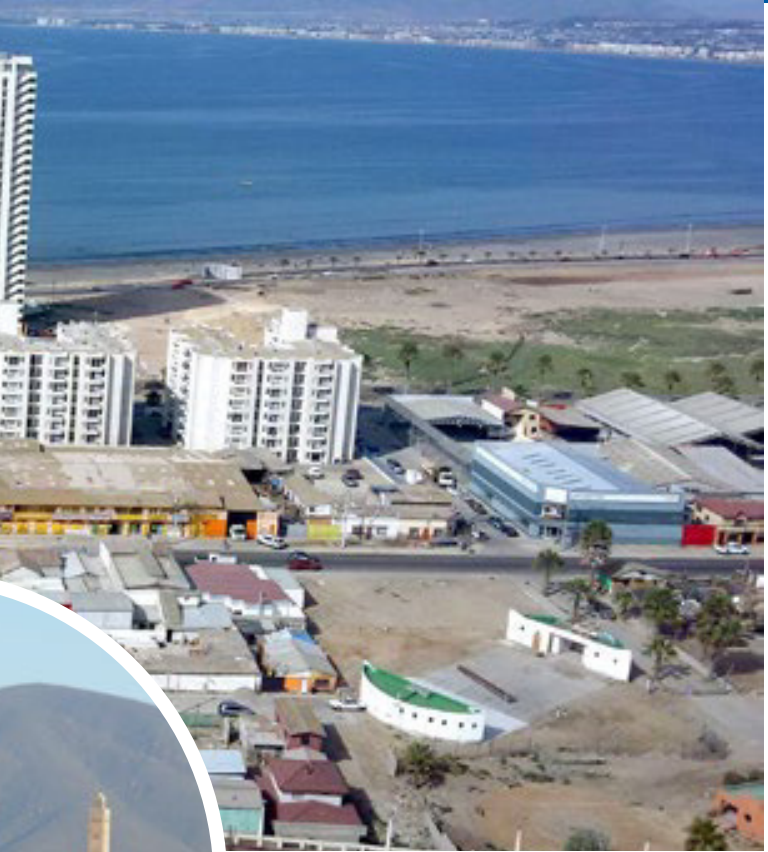

existen variables externas en donde están expuestas las empresas constructoras lo que afectaría tanto en sus duraciones como en sus costos.

Dichas variables son; condición de mercado del recurso humano, insumos o materia prima, así como también la condición climática de la región.

En el presente estudio proponemos determinar una función de ajuste $K$ a ser aplicada a la programación de la duración de las actividades con el fin de buscar una mayor precisión entre las duraciones programadas y las duraciones reales; lo anterior basado en la proposición realizada en [2].

Por lo tanto se propone determinar la función:

En donde:

$$
K=f(c, t, i)
$$

$K$ es el factor de ajuste a la programación de las duraciones

$c$ es el porcentaje de variación de las condiciones climáticas a considerar en la región o localidad; $t$ es el porcentaje de variación de la tasa de cambio de la moneda US\$, respecto a un año determinado; $i$ es el porcentaje de crecimiento del desarrollo industrial regional o nacional, según sea el o los índices a considerar.

Para nuestro estudio se consideró que para la variable Condiciones Climáticas, lo más importante es la temperatura media y el porcentaje de humedad. Para la variable Desarrollo Industrial, consideramos el PIB base a precios del año 2008, así como la Tasa Regional de Desempleo. Finalmente para la variable Tasa de Cambio se tomó como referencia el año 2008.

Se trataron los datos en términos trimestrales desde el primer trimestre del año 2009 al segundo trimestre del año 2013. El resumen de los datos anteriormente indicados, se encuentran en la Tabla 3 siguiente. 
Tabla 3. Resumen de Datos de Clima, Tasa de Cambio y Desarrollo Industrial

\begin{tabular}{|c|c|c|c|c|c|c|c|c|}
\hline Año & Trimestre & $\mathrm{T}^{0}$ MEDIA & $\begin{array}{c}\text { \% Humedad } \\
\text { Relativa }\end{array}$ & Dólar (Ch\$) & Dólar (\%) & $\begin{array}{l}\text { Desempleo } \\
\text { (miles pers) }\end{array}$ & $\begin{array}{c}\text { Desempleo } \\
(\%)\end{array}$ & $\begin{array}{c}\text { PIB nac } \\
(\%)\end{array}$ \\
\hline \multirow{4}{*}{2009} & Dic - Feb & 16,7 & \begin{tabular}{|l|}
76,8 \\
\end{tabular} & 626,1 & $3,60 \%$ & 21,5 & $8,00 \%$ & $-2,87 \%$ \\
\hline & Mar - May & 14,1 & 83,7 & 580,6 & $-7,27 \%$ & 21,2 & $8,17 \%$ & $2,40 \%$ \\
\hline & Jun - Ago & 10,5 & 82,6 & 546,8 & $-5,82 \%$ & 24,7 & $9,02 \%$ & $1,39 \%$ \\
\hline & Sept - Nov & 12,7 & 77,7 & 534,2 & $-2,30 \%$ & 21,2 & $7,57 \%$ & $12,57 \%$ \\
\hline \multirow{4}{*}{2010} & Dic - Feb & 15,9 & 78,8 & 511,6 & $-4,24 \%$ & 17,3 & $5,93 \%$ & $-4,16 \%$ \\
\hline & Mar - May & 13,9 & 82,3 & 525,7 & $2,76 \%$ & 27,6 & $9,17 \%$ & $5,02 \%$ \\
\hline & Jun - Ago & 9,9 & 81,1 & 525,9 & $0,05 \%$ & 24,1 & $7,58 \%$ & $4,34 \%$ \\
\hline & Sept - Nov & 12,6 & 78,7 & 486,8 & $-7,44 \%$ & 22,8 & $7,28 \%$ & $9,64 \%$ \\
\hline \multirow{4}{*}{2011} & Dic - Feb & 16,5 & 76,3 & 480,0 & $-1,39 \%$ & 26,7 & $8,38 \%$ & $-2,27 \%$ \\
\hline & Mar - May & 13,7 & 79,9 & 472,9 & $-1,47 \%$ & 27,2 & $8,36 \%$ & $-1,44 \%$ \\
\hline & Jun - Ago & 10,5 & 81,9 & 466,4 & $-1,38 \%$ & 24,6 & $7,45 \%$ & $-0,47 \%$ \\
\hline & Sept - Nov & 12,7 & 80,0 & 501,3 & $7,49 \%$ & 19,5 & $5,99 \%$ & $10,69 \%$ \\
\hline \multirow{4}{*}{2012} & Dic - Feb & 17,3 & 75,5 & 500,0 & $-0,26 \%$ & 23,2 & $6,69 \%$ & $-3,02 \%$ \\
\hline & Mar - May & 14,7 & 79,7 & 489,5 & $-2,10 \%$ & 19,6 & $5,73 \%$ & $0,98 \%$ \\
\hline & Jun - Ago & 10,8 & 78,1 & 492,9 & $0,68 \%$ & 19,5 & $5,83 \%$ & $0,07 \%$ \\
\hline & Sept - Nov & 13,1 & 77,0 & 477,0 & $-3,22 \%$ & 21,7 & $6,32 \%$ & $10,26 \%$ \\
\hline \multirow{2}{*}{2013} & Dic - Feb & 17,5 & 76,8 & 474,1 & $-0,61 \%$ & 19,1 & $5,70 \%$ & $-4,16 \%$ \\
\hline & Mar - May & 13,7 & 78,5 & 474,7 & $0,15 \%$ & 24,8 & $7,43 \%$ & $-0,27 \%$ \\
\hline
\end{tabular}

Fuentes: Dirección Meteorológica de Chile, Banco Central de Chile e Instituto Nacional de Estadística de Chile. 2012
Realizando el estudio respectivo de estas variables, se determinan y se seleccionan y se propone la función $K$ de ajuste. La cual queda de la siguiente forma:

$$
K=f(t m, h, t, d, p)
$$

En donde:

$K$ es el factor de ajuste a la programación de las duraciones

tm es la temperatura media de la zona o región

$h$ es el porcentaje de humedad relativa de la zona

$t$ es el porcentaje de variación de la tasa de cambio de la moneda americana, respecto a un año determinado

$d$ es el porcentaje de desempleo de la región

$p$ es el porcentaje de crecimiento del PIB en la industria de la construcción

Con los datos de la Tabla 3 y los antecedentes de las desviaciones obtenidas en la duración de los proyectos más representativos de la conurbación Coquimbo-La Serena, se conforma un modelo de regresión lineal, como se muestran en la Tabla 4.

Tabla 4. Principales Proyectos de Edificios en la Región de Coquimbo

\begin{tabular}{|c|c|c|c|c|c|c|c|}
\hline \multirow{2}{*}{ edificio } & \multirow{2}{*}{$\begin{array}{c}\text { Fecha Inicio } \\
\text { Real } \\
\end{array}$} & \multicolumn{2}{|c|}{ Fecha Término } & \multicolumn{2}{|c|}{ duracion (dias) } & \multirow{2}{*}{$\begin{array}{c}\text { diferencia } \\
\text { (dias) }\end{array}$} & \multirow{2}{*}{$\begin{array}{c}\text { diferencia } \\
\%\end{array}$} \\
\hline & & programada & real & programada & real & & \\
\hline Edificio Altamar & dic-09 & mar-11 & $a b r-11$ & \begin{tabular}{|l|}
439 \\
\end{tabular} & 470 & \begin{tabular}{|l|}
31 \\
\end{tabular} & 7,06 \\
\hline Brisas de San Joaquin & $a b r-12$ & oct-12 & dic-12 & 153 & 233 & 80 & 52,29 \\
\hline Espacio Urbano II & may-12 & mar-13 & jul-13 & 214 & 310 & 96 & 44,86 \\
\hline Paseo San Carlos & may-12 & ene-13 & $a b r-13$ & 180 & 230 & 50 & 27,78 \\
\hline Condominio Mistral III & feb-12 & ene-13 & may-13 & 260 & 331 & 71 & 27,31 \\
\hline
\end{tabular}

Fuente: Elaboración Propia

En base al modelo propuesto, la función $K$ quedaría definida de la siguiente forma [5]:

$$
K=a_{1}+a_{2} t m+a_{3} h+a_{4} t+a_{5} d+a_{6} i
$$

$a_{1} a_{2}, a_{3}, a_{4}, a_{5}$ y $a_{6}$ son los coeficientes de la función

En' base a la disponibilidad de valores de desviación obtenidas de las duraciones de los proyectos, se considerará los antecedentes de las variables dependientes propuestas a partir del año 2011 en adelante. Por otra parte, al realizar una determinación como la propuesta, no se obtiene un resultado positivo, ya que se propuso hacer un modelo exponencial. Sin embargo al tener valores negativos en los datos, no es posible la linealización de la función. Entonces, para bajar la negatividad de los datos, éstos se manejan en términos cuadráticos y se prueba un modelo regresivo lineal múltiple, encontrándose con esto la respuesta esperada. Para todos los cálculos se utiliza el Complemento de Excel (MS ${ }^{\mathrm{TM}}$ ) Análisis de Datos.

En la Tabla 5 se muestra la matriz con los datos en términos cuadráticos considerada para el cálculo.
Tabla 5. Matriz de datos en términos cuadráticos

\begin{tabular}{|r|r|r|r|r|r|}
\hline K & \multicolumn{1}{|c|}{ T } & \multicolumn{1}{|c|}{ H } & \multicolumn{1}{|c|}{ Tc } & \multicolumn{1}{|c|}{ Ds } & \multicolumn{1}{c|}{ Pib } \\
\hline 0,0049865 & 278,89 & 5894,82716 & 0,001296 & 0,00639357 & 0,00082369 \\
\hline 0,0049865 & 199,751111 & 7000,11111 & 0,00528106 & 0,00668278 & 0,000576 \\
\hline 0,0049865 & 110,25 & 6815,41975 & 0,00339296 & 0,00813817 & 0,00019321 \\
\hline 0,0049865 & 160,444444 & 6032,11111 & 0,00052764 & 0,00573026 & 0,01580049 \\
\hline 0,0049865 & 253,871111 & 6205,93827 & 0,00180072 & 0,00351735 & 0,00173056 \\
\hline 0,0049865 & 192,284444 & 6778,77778 & 0,00075971 & 0,00841767 & 0,00252004 \\
\hline 0,0049865 & 98,6711111 & 6579,01235 & $2,0846 \mathrm{E}-07$ & 0,0057502 & 0,00188356 \\
\hline 0,0049865 & 157,921111 & 6188,44444 & 0,00553905 & 0,00530545 & 0,00929296 \\
\hline 0,0049865 & 271,151111 & 5826,77778 & 0,00019459 & 0,00701421 & 0,00051529 \\
\hline 0,0049865 & 187,69 & 6382,23457 & 0,00021698 & 0,00699399 & 0,00020736 \\
\hline 0,0049865 & 110,951111 & 6705,79012 & 0,00019009 & 0,0055491 & 0,00002209 \\
\hline 0,0049865 & 160,444444 & 6400 & 0,00560299 & 0,00358604 & 0,01142761 \\
\hline 0,0049865 & 300,4444444 & 5701,06186 & $6,6222 \mathrm{E}-06$ & 0,004447495 & 0,00091204 \\
\hline 0,0049865 & 215,111111 & 6353,63267 & 0,000441 & 0,00328059 & 0,00009604 \\
\hline 0,2734244 & 115,921111 & 6105,26586 & $4,6836 \mathrm{E}-05$ & 0,00340302 & 0,00000049 \\
\hline 0,201242 & 172,4844444 & 5930,65603 & 0,00103818 & 0,00399167 & 0,01052676 \\
\hline 0,0771728 & 306,25 & 5901,95912 & $3,7479 \mathrm{E}-05$ & 0,0032456 & 0,00173056 \\
\hline 0,0745836 & 188,604444 & 6164,43827 & $2,1186 \mathrm{E}-06$ & 0,00552147 & 0,00000729 \\
\hline
\end{tabular}

Fuente: Elaboración propia 
Tabla 6. Resultados del ajuste

\begin{tabular}{|c|c|c|c|c|c|c|c|c|}
\hline Resumen & & & & & & & & \\
\hline \multicolumn{9}{|l|}{ Estadísticas de la regresión } \\
\hline Coeficiente de correlación múltiple & 0,9484 & & & & & & & \\
\hline Coeficiente de determinación $\mathrm{R}^{\wedge} 2$ & 0,8995 & & & & & & & \\
\hline $\mathrm{R}^{\wedge} 2$ ajustado & 0,7738 & & & & & & & \\
\hline Error típico & 0,0459 & & & & & & & \\
\hline Observaciones & 10 & & & & & & & \\
\hline \multicolumn{9}{|l|}{ ANÁLISIS DE VARIANZA } \\
\hline & $\begin{array}{c}\text { Grados de } \\
\text { libertad }\end{array}$ & $\begin{array}{c}\text { Suma de } \\
\text { cuadrados }\end{array}$ & $\begin{array}{l}\text { Promedio } \\
\text { de los } \\
\text { cuadrados }\end{array}$ & $F$ & $\begin{array}{c}\text { Valor crítico } \\
\text { de F }\end{array}$ & & & \\
\hline Regresión & 5 & 0,0754 & 0,0151 & 7,157 & 0,0399 & & & \\
\hline Residuos & 4 & 0,0084 & 0,0021 & & & & & \\
\hline \multirow[t]{3}{*}{ Total } & 9 & 0,0838 & & & & & & \\
\hline & & & & & & & & \\
\hline & Coeficientes & Error típico & Estadistico $t$ & Probabilidad & Inferior 95\% & Superior 95\% & Inferior $95,0 \%$ & Superior $95,0 \%$ \\
\hline Intercepción & 2,232 & 0,599 & 3,728 & 0,020 & 0,570 & 3,893 & 0,570 & 3,893 \\
\hline Variable X 1 & $-0,0015$ & 0,000 & $-4,328$ & 0,012 & $-0,003$ & $-0,001$ & $-0,003$ & $-0,001$ \\
\hline Variable X 2 & $-0,0003$ & 0,000 & $-3,189$ & 0,033 & $-0,001$ & 0,000 & $-0,001$ & 0,000 \\
\hline Variable $\times 3$ & $-22,508$ & 17,976 & $-1,252$ & 0,279 & $-72,418$ & 27,401 & $-72,418$ & 27,401 \\
\hline Variable X 4 & $-24,655$ & 11,194 & $-2,203$ & 0,092 & $-55,733$ & 6,424 & $-55,733$ & 6,424 \\
\hline Variable X 5 & 2,803 & 6,949 & 0,403 & 0,707 & $-16,492$ & 22,097 & $-16,492$ & 22,097 \\
\hline
\end{tabular}

Fuente: Elaboración propia

Sobre la base de los resultados obtenidos, se propone el modelo que determina el valor de $K$ en términos cuadráticos y así obtener el valor de $K$ final [6].

$\mathrm{K}^{2}=\mathrm{a}_{1}+\mathrm{a}_{2} \mathrm{tm}^{2}+\mathrm{a}_{3} \mathrm{~h}^{2}+\mathrm{a}_{4} \mathrm{t}^{2}+\mathrm{a}_{5} \mathrm{~d}^{2}+\mathrm{a}_{6} \mathrm{i}^{2}$

$K^{2}=2.232-0.0015 t m^{2}-0.0003 h^{2}-22.508 t^{2}-24.655 d^{2}+2.803 i^{2}$

$K=\sqrt{2.232-0.0015 t m^{2}-0.0003 h^{2}-22.508 t^{2}-24.655 d^{2}+2.803 i^{2}}$

De (6) y reemplazando los valores de la Tabla 5, se obtiene:

$$
K=0.27
$$

Al obtener el valor $K$, interpretamos que a la programación de la duración de las actividades de un proyecto es necesario agregar un $27 \%$ para su proposición final. A modo de prueba, se consideró la programación de los proyectos en estudio, aplicando el Factor K. Los resultados se aprecian en la Tabla 7.

Tabla 7. Duraciones de las actividades de los proyectos, utilizando Factor $K$

\begin{tabular}{|c|c|c|c|c|c|c|c|c|c|c|}
\hline \multirow{2}{*}{$\mathrm{N}^{\circ}$} & \multirow{2}{*}{ edificio } & \multirow{2}{*}{$\begin{array}{c}\text { Fecha Inicio } \\
\text { Real }\end{array}$} & \multicolumn{2}{|c|}{ Fecha Término } & \multicolumn{2}{|c|}{ duracion (dias) } & \multirow{2}{*}{$\begin{array}{c}\text { diferencia } \\
\text { (dias) }\end{array}$} & \multirow{2}{*}{$\begin{array}{c}\text { diferencia } \\
(\%)\end{array}$} & \multirow{2}{*}{$\begin{array}{l}\text { Programada } \\
\text { con K (dias) }\end{array}$} & \multirow{2}{*}{$\begin{array}{l}\text { diferencia } \\
\text { con K ( \% ) }\end{array}$} \\
\hline & & & programada & real & programada & real & & & & \\
\hline 1 & $\begin{array}{l}\text { Edificio } \\
\text { Altamar }\end{array}$ & dic-09 & mar-11 & abr-11 & 439 & 470 & 31 & 7,06 & 557,5 & $15,70 \%$ \\
\hline 2 & $\begin{array}{c}\text { brisas de sn } \\
\text { joaquin }\end{array}$ & may-12 & oct-12 & dic-12 & 153 & 233 & 80 & 52,29 & 194,3 & $-19,91 \%$ \\
\hline 3 & $\begin{array}{l}\text { Espacio } \\
\text { Urbano II }\end{array}$ & may-12 & mar-13 & jul-13 & 214 & 310 & 96 & 44,86 & 271,8 & $-14,06 \%$ \\
\hline 4 & $\begin{array}{c}\text { Paseo San } \\
\text { Carlos }\end{array}$ & may-12 & ene-13 & abr-13 & 180 & 230 & 50 & 27,78 & 228,6 & $-0,61 \%$ \\
\hline 5 & $\begin{array}{c}\text { Condominio } \\
\text { Mistral III }\end{array}$ & feb-12 & ene-13 & may-13 & 260 & 331 & 71 & 27,31 & 330,2 & $-0,24 \%$ \\
\hline
\end{tabular}

Fuente: Elaboración propia 
De los resultados anteriores vistos en la Tabla 7, se desprende que si se hubiera considerado el Factor $K$ en la programación de los proyectos considerados, las desviaciones claramente se hubieran visto reducidas de manera significativa, lo que habría significado reducir el impacto en los costos de las inversiones y las empresas, hubieran podido aprovechar mejor desde el punto de vista económico, las oportunidades del mercado, así como la posibilidad de poder ofrecer un precio más competitivo por sus productos.

\section{CONCLUSIONES.}

Una vez realizado los cálculos para la determinación del Factor $K$ de ajuste a las duraciones de las actividades de un proyecto de construcción de un edificio en altura, podemos establecer que las variables consideradas inicialmente como cualitativas, Se pueden manejar en términos cuantitativos.

Al utilizar el Factor $K$ de ajuste se pudo dar cuenta que al tener presente la aplicación de dicho factor obtenemos una importante baja en el porcentaje de desviación de las duraciones. El efecto más notorio se observa en los dos últimos proyectos, en los cuales se obtiene solo una variación de un día. Esto nos lleva a pensar que las variaciones se presentan según las condiciones dadas o la evolución que tengan las variables incluidas en el modelo. De esta manera será posible bajar tanto la incerteza como la desviación de la duración de los proyectos, lo que traería consigo que los inversionistas colocaran sus productos al mercado en el momento que se necesiten; además de reducir los riesgos del incremento de los costos financieros asociados al financiamiento del proyecto [7].

Es muy posible que los ajustes a la programación de proyectos en cuanto a su duración sean explicados también por otras variables internas al proyecto. Las variables que desprenden del análisis de los resultados obtenidos del modelo, podrían ser expertos del programador, grado de capacitación del personal del proyecto o que pueden existir otras variables externas que también incidan y que es importante considerar al momento de realizar un proyecto de estas características para llegar a su término con una desviación mínima, que es lo esperado por sus dueños o inversionistas.

Finalmente, para continuar con este estudio, se propone continuar buscando variables que incidan de forma interna o externa a los proyectos y su cuantificación, tanto en las duraciones como en los costos, utilizando herramientas tanto matemáticas como tecnológicas simples que puedan ser aplicadas por cualquier profesional del área.

\section{BIBLIOGRAFÍA}

1) W. S. Herroelen. "Project Scheduling-Theory and Practice", Production and Operations Management; Vol. 14, No. 4, Winter 2005, p. 413-432.

2) O. Contreras G. "Aplicación y Análisis del Modelo Pert/ Cpm Modificado, en la Programación de la Duración de las Actividades en la Construcción de Edificios en Altura en Coquimbo, Chile", Iberoamerican Journal of Project Management, Vol. 3, N¹, 2012.

3) M. Ishaque, A. K. Zaidi, A. H. Levis. "Project Management Using Point Graphs", Systems Engineering, Vol. 12, No. 1, 2009, p. 3654.

4) S. Capuz, E. Gómez-Senent, A. Torrealba López; P. Ferrer Gisbert, J. L. Vivancos, T. Gómez. "Dirección, Gestión y Organización de Proyectos", Cuadernos de Ingeniería de Proyectos III; Editorial UPV, España, 2002.

5) N. Gujarati, Damodar, C. Porte Dawn. "Econometría”, 2010, 5ta Ed., Editorial McGraw Hill, Cap. 6.

6) H. Kohler. "Estadística para negocios", 1996, 1ra Ed., Editorial CECSA, Cap. 13.

7) M.Vanhoucke, A. Vereecke, P. Gemmel. "The Project Scheduling Game (PSG): Simulating Time/Cost Trade-Offs in Projects", The Project Management Institute, 2005, Vol. 36, No. 1, 51-59, ISSN 8756-9728/03.

8) A. Serpell, L. Alarcón. "Planificación y Control de Proyectos", 2003, 2da. Ed., Editorial Universidad Católica de Chile.

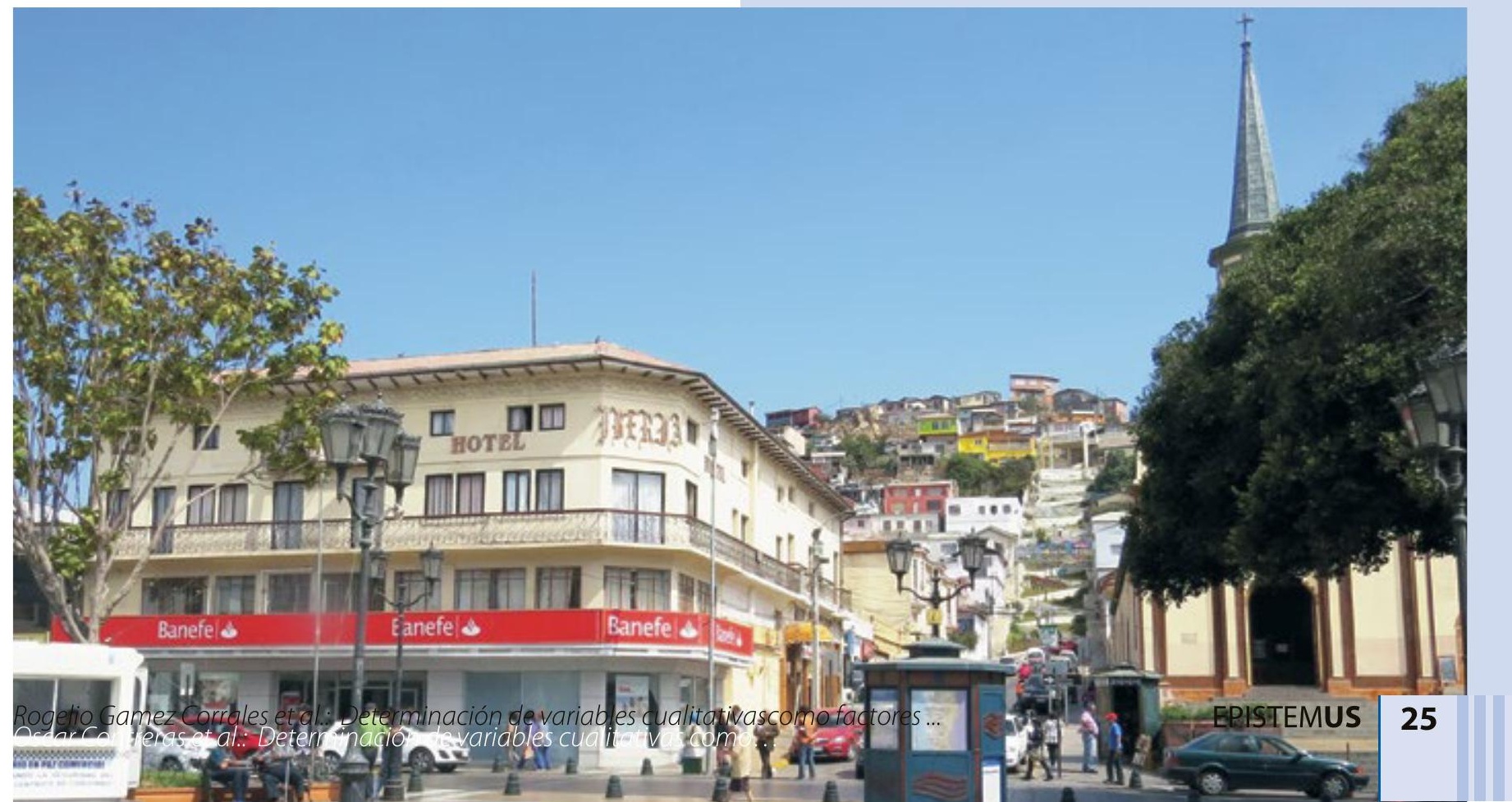

